\title{
Multi-objective Optimisation of Web Business Processes
}

\author{
Ashutosh Tiwari, Christopher Turner, Peter Ball and Kostas Vergidis \\ Decision Engineering Centre, Cranfield University, Cranfield, Bedfordshire, UK, MK43 0AL \\ \{a.tiwari, c.j.turner, p.d.ball\}@ cranfield.ac.uk
}

\begin{abstract}
This paper proposes an approach for the optimisation of web business processes using multi-objective evolutionary computing. Business process optimisation is considered as the problem of constructing feasible business process designs with optimum attribute values such as duration and cost. This optimisation framework involves the application of a series of Evolutionary Multi-objective Optimisation Algorithms (EMOAs) in an attempt to generate a series of diverse optimised business process designs for given requirements. The optimisation framework is tested to validate the framework's capability in capturing, composing and optimising business process designs constituted of web services. The results from the web business process optimisation scenario, featured in this paper, demonstrate that the framework can identify business process designs with optimised attribute values.
\end{abstract}

Keywords: Multi-objective optimisation, Business Process, EMOA, Web services

\section{$1 \quad$ Introduction}

The design and management of business processes is a key factor for companies to effectively compete in today's volatile business environment. By focusing on the optimisation and continuous improvement of business processes, organisations can establish a solid competitive advantage by reducing cost, improving quality and efficiency, and enabling adaptation to changing requirements. This paper discusses how a business process optimisation framework $\left(\mathrm{bpo}^{\mathrm{F}}\right.$ ), based on Evolutionary Multiobjective Optimisation Algorithms (EMOAs), can be applied to the real-world optimisation of actual web business processes. The details of $\mathrm{bpo}^{\mathrm{F}}$ are presented in [1]. The proposed optimisation framework is tested with a web business process scenario to validate the framework's capability in capturing, composing and optimising process designs constituted of web services.

According to Davenport and Short [2] a business process is a set of logically related tasks performed to achieve a defined business outcome. A business process is perceived as a collective set of tasks that when properly connected perform a business operation. A web business process is therefore a process composed of web services, where the function each process task performs is enacted by an individual web service. A web service can be thought of as 'a discretely defined set of contiguous and 
autonomous business or technical functionality implemented over a network' [1]. A further discussion of the literature for business process optimisation can be found in [3]. There are limited approaches for optimising business processes. Such approaches can only deal with simple sequential business processes within a single objective optimisation framework [4]. The work of Ko et al. [5] highlights a number of examples of soft computing use in the business process arena.

\section{Quantitative Representation of Business Process Designs}

The business process optimisation framework $\left(\mathrm{bpo}^{\mathrm{F}}\right)$, applied here, is described in detail in [1]. The main elements involved are the tasks and resources of the business process. The attributes of the tasks and the process are also taken into consideration in order to provide the capability of evaluating a business process design. Finally, the patterns that interconnect the tasks are also included. As task attributes, we consider measurable (quantitative) characteristics of the tasks. Examples involve task cost and task duration. The task attributes can be mapped to the corresponding process attributes (e.g. process cost) using a suitable aggregate function. We consider the inputs and outputs of a task as task resources. The task resources connect the various tasks based on their inputs and outputs. Also, the resources provide the requirements for a process design in the form of required process input and process output.

The chromosome of each individual is made up of task numbers which are then organised into a specific sequence by the Process Composition Algorithm (PCA). It is the PCA that creates the process graphs out of the tasks provided in an individual. Both crossover and mutation operators are used in the manipulation of individuals. The task library can be used to help repair an individual by the addition of extra tasks as that individual is built into a process by the PCA. The general parameter settings for the EMOAs used are shown in Table 1. The main steps of the proposed business process optimisation framework are detailed in [6].

\section{Web Business Process Optimisation}

The scenario featured in this paper describes an automated sales forecasting process (scenario provided by [7]). This process is considered as semi-automated as it involves the interaction of some applications but it is not streamlined and still requires human involvement in the act of generating and visualising the requested forecasts. In this process each of the tasks (marked 1- 5 in Fig. 1) is a web service carrying out a unique function, such as generating graphs etc. The aim of this scenario is to show the optimisation potential of the framework. Fig. 1 shows the generic business process design for the scenario. It involves two input resources: (a) company name and (b) market update request. The first resource is necessary for the web service to extract relevant data for the specified company. The second resource is a request for a market update that needs to be considered for the sales forecast. 


\subsection{Library of tasks (web services)}

Having sketched the initial business process design, we can compile the library of alternative web services based on the main steps of the generic process design.

Table 1. General parameters

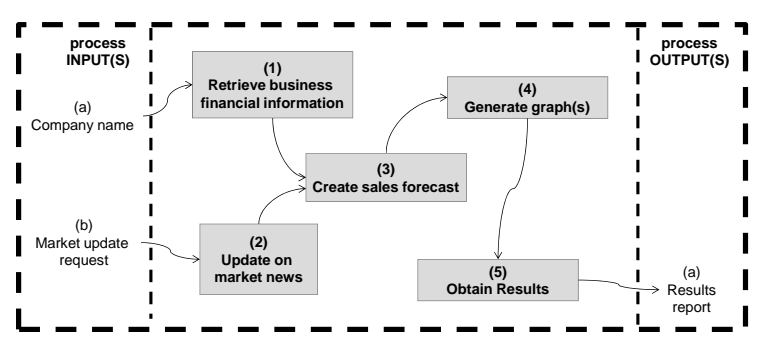

\begin{tabular}{|l|l|}
\hline Population & 250 \\
\hline Generations & 25,000 \\
\hline $\begin{array}{l}\text { Crossover } \\
\text { probability }\end{array}$ & 0.8 \\
\hline $\begin{array}{l}\text { Mutation } \\
\text { probability }\end{array}$ & 0.2 \\
\hline Objectives & 2 \\
\hline
\end{tabular}

Fig. 1. Initial business process design for sales forecasting

Relevant research on the selected on-line libraries of web services resulted in a selection of 20 web services from different providers that can potentially contribute to implementing the scenario (an example table of web services can be found in [1] $\mathrm{p}$. 213). Each task in the scenario has input and output resources (an example of the table of resources can be found in [1] p. 212). The proposed optimisation framework is tested for two objectives; Service Delivery Price (SDP) (specifies the amount of money the service customer has to pay for the consumption of distinct service volumes, i.e. the cost to use the service) and Service Fulfilment Target (SFT) (specifies the service provider's promise of effective and seamless delivery of the defined benefits to any authorised service consumer). SFT is expressed as the promised maximum number of successful individual service deliveries with respect to the total counts of individual service deliveries.

Having gathered all the necessary information (library of web services and input/output resources for each web service), the problem parameters can be defined based on the business process problem formulation (an example of parameter values can be found in [6]). This results in a complete scenario ready to be tested within the proposed optimisation framework.

\subsection{Generate the scenario's search space}

The first step towards visualising the results is to generate the scenario's search space by producing 1000 random feasible process designs. The initial business process design in Fig. 1 involves 5 main steps. A design with less than 5 tasks shows that there is a web service that consolidates two or more tasks. A design with more tasks shows that one step requires two or more web services to be implemented. The search space for this scenario is shown in Fig. 2. The search space consists of five different regions, each corresponding to a group of designs with same number of tasks $(4,5,6$, 7 or 8$)$ 


\subsection{Test the scenario with bpo ${ }^{\mathrm{F}}$}

The challenge for the EMOAs in the framework is to identify non-dominated (optimised) solutions in each of the regions in the search space. Fig. 2 shows the results obtained by combining the outputs of four EMOA algorithms (the four algorithms used were NSGA2 (Non-dominated Sorting Genetic Algorithm 2), PAES (Pareto Archived Evolutionary Strategy), PESA2 (Pareto Envelope-based Selection Algorithm 2) and SPEA2 (Strength Pareto Evolutionary Algorithm 2)). All the algorithms identify process designs near the Pareto front of each of the five regions of the search space. This is a strong indicator of the performance of the algorithms and the confidence in the generated designs being near optimal. Fig. 2 demonstrates two optimised business process designs (a) and (b), one with 4 tasks and one with 6 tasks (taken from the Pareto fronts indicated in Fig. 2). Each of these designs belongs to a different island based on its size. The arrows in Fig. 2 indicate the island from where each design in Fig. 3 originates. Fig.3. (a) shows a business process design with one of the generic steps missing. The forecasting results are not plotted into a graph but they are just faxed back to the requestor. The framework reduces cost in this instance. Therefore, in a semi-automated process the framework can take 'initiative' and alter the generic design provided that the process input and output requirements are still satisfied. Fig. 3(b) is composed of 6 services and involves two tasks for obtaining the company's financial data either from selecting one or both (OR is not exclusive choice). This provides better confidence in terms of accuracy of the data obtained and improved reliability of the process execution itself.

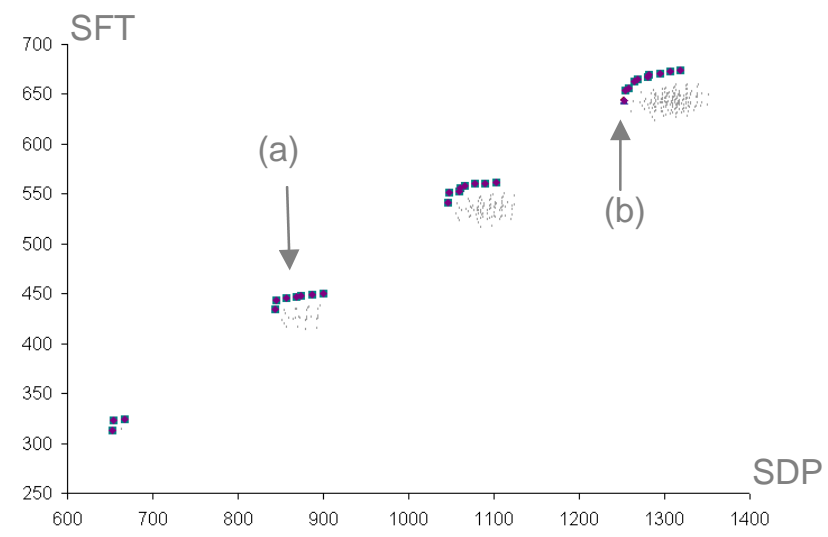

Fig. 2. Search space and EMOA results for the scenario

\section{Conclusions}

The scenario featured in this paper demonstrated how an optimised business process can be automatically created by the optimisation framework using web services. The framework identified the optimal designs for all the available process sizes. The generated designs select and incorporate different web services arranged with the 
appropriate process patterns so that (i) the process input and output requirements are satisfied and (ii) the attribute values are optimised. Results from the real-life scenario, featured in this paper, demonstrate that the optimisation framework can identify business process designs with optimised attribute values.

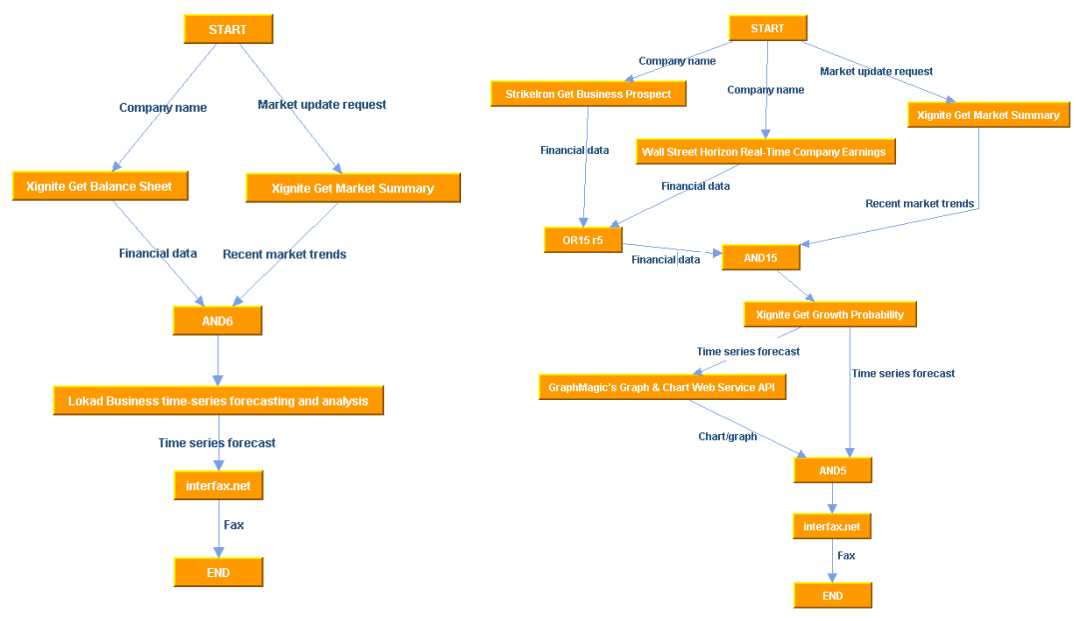

Fig. 3. Optimised business process designs for the scenario (process a (on the left) has 4 tasks and process $b$ (on the right) has 6 tasks)

\section{References}

[1] Vergidis, K.: Business Process Optimisation Using an Evolutionary Multi-objective Framework, PhD Thesis, School of Applied Sciences, Cranfield University, Cranfield, Bedfordshire, UK (2008)

[2] Davenport, T.H. and Short, J.E.: The New Industrial Engineering: Information Technology and Business Process Redesign, Sloan Management Review, Summer 1990, pp. 11-27 (1990)

[3] Vergidis, K.; Tiwari, A.; Majeed, B.: Business Process Analysis and Optimization: Beyond Reengineering. Systems, Man, and Cybernetics, Part C: Applications and Reviews, IEEE Transactions on, vol.38, no.1, pp.69-82 (2008)

[4] Hofacker, I. and Vetschera, R.: Algorithmical Approaches to Business Process Design, Computers \& Operations Research, Vol. 28, pp. 1253-1275 (2001)

[5] Ko, M., Tiwari, A., and Mehnen, J.: A Review of Soft Computing Applications in Supply Chain Management, Applied Soft Computing, 10(3), pp. 661-674 (2010)

[6] Tiwari, A., Vergidis, K., and Turner, C.J.: Evolutionary Multi-Objective Optimisation of Business Processes, In: Gao, X.Z., Gaspar-Cunha, A., Köppen, M., Schaefer, G., Wang, J. (eds.) Advances in Intelligent and Soft Computing: Soft Computing in Industrial Applications, Springer, Heidelberg, Germany, In press (2010)

[7] Grigori, D., Casati, F., Castellanos, M., Dayal, U., Sayal, M. and Shan, M.-C.: Business Process Intelligence, Computers in Industry, vol. 53, pp. 321-343 (2004) 